\title{
Double-Blind Single-Session Neurofeedback Training in Upper-Alpha for Cognitive Enhancement of Healthy Subjects
}

\author{
C. Escolano \\ B. Olivan \\ Y. Lopez-del-Hoyo \\ J. Garcia-Campayo \\ J. Minguez
}

\begin{abstract}
This paper reports on a single-session neurofeedback (NF) training procedure on the user-specific upper alpha band for cognitive enhancement in healthy users. A doubleblind study was designed using a NF group and an active control group. Control group performed as the NF group but received sham feedback, minimizing the non-specific factors of training. This design aimed to $(i)$ investigate upper alpha as a NF parameter, $(i i)$ evaluate the NF effects on upper alpha during the execution of a cognitive task, and (iii) evaluate the effects on cognitive performance by means of a cognitive task and a battery of psychological tests. Results of EEG analysis show the key role of the feedback: only the NF group enhanced upper alpha during the training, and it led to a desynchronization increase during the execution of the cognitive task. Regarding the behavioral results, a strong learning effect was observed, with the NF group performing better in almost all measurements but many of them without statistical significance.
\end{abstract}

\section{INTRODUCTION}

The electroencephalogram (EEG) measures brain electrical activity with sensors placed over the surface of the scalp, providing information on the underlying brain oscillations. Nowadays, research on the interpretation of the functional significance of these oscillations is a trend topic in neuroscience. Some integrative hypothesis have been proposed and the correlation of brain oscillations with brain functioning is accepted [1], [2]. Alpha activity has been traditionally linked to cognitive performance [3]. Alpha rhythm is the dominant frequency in the human EEG and is characterized by a peak in the range [7.5-12.5] $\mathrm{Hz}$ [3]. It has been recently hypothesized that alpha rhythm may act in the cortex as a mechanism to inhibit unnecessary or conflicting processes to the task being performed, thus facilitating attention by actively suppressing distracting stimuli [4].

In this sense, neurofeedback (NF) has emerged as a potential technique to allow users to modulate their brain rhythms using an operant control paradigm, thus altering brain functioning. The basic principle of NF consists of measuring brain activity, decoding or identifying the brain patterns of interest, and then providing the user with feedback stimuli depending on the desired working levels of these rhythms.

Carlos Escolano and Javier Mínguez are with the Instituto de Investigación en Ingeniería de Aragón (I3A) and the Universidad de Zaragoza, Spain. Javier Minguez is also with Bit\&Brain Technologies SL, Spain. E-mail: \{cescolan, jminguez\}@unizar.es. Bárbara Oliván Blázquez and Yolanda López del Hoyo are with the Department of Psychology and Sociology, University of Zaragoza, Spain. E-mail: \{bolivan, yolandal $\} @$ unizar.es. Javier García-Campayo is with the Department of Psychiatry and Miguel Servet University Hospital, University of Zaragoza, Spain. E-mail: jgarcamp@gmail.com. This work has been supported by projects HYPERCSD2009-00067 and DPI2009-14732-C02-01 of the Spanish Government, and by DGA-FSE (grupo T04)
Several NF training studies have reported therapeutic effects in a variety of neurological and psychological disorders such as epilepsy, attention deficit hyperactivity disorder (ADHD), and addictive disorders, among others (see [2] for a review). Furthermore, NF training applied to healthy users has demonstrated to improve certain cognitive aptitudes [5]-[8].

Some alpha-based NF training approaches adjust the alpha frequency per user instead of using a fixed band, and divide alpha band into two narrower sub-bands since they selectively respond to different task demands [3]. These approaches deal with the inherent high inter-user variability of alpha, and minimize the unspecific behavioral effects of training the entire alpha band. Alpha can be individually adjusted using the Individual Alpha Frequency (IAF), which is an anchor point that distinguishes between the upper and the lower alpha sub-bands, being related the former one to cognitive performance [3]. Examples include a single-session study that reported cognitive enhancement in a mental rotation task applying upper alpha NF training [7], and these results were also extended to a five-session study [5], [6].

This study reports on a single-session NF training procedure on the upper alpha frequency band, applied to healthy users for cognitive enhancement. It has been designed in a double-blind fashion using a NF training group and an active control group, where the control group performed as the NF group but received sham feedback (placebo). This design minimized the non-specific factors of training (commonly criticized in NF literature [8]). The main objectives were to ( $i$ ) investigate upper alpha as a NF parameter, $(i i)$ evaluate the NF effects on upper alpha band during the execution of a cognitive task, and ( $i i i)$ evaluate the effects on cognitive performance by means of a cognitive task and a battery of psychological tests. Results of EEG analysis show the key role of the feedback: only the NF group enhanced upper alpha during the training, and it led to a desynchronization increase during the execution of the cognitive task. Regarding the behavioral results, a strong learning effect was observed, with the NF group performing better in almost all measurements but many of them without statistical significance.

\section{MethodS}

\section{A. Participants and Experimental Setup}

Nineteen subjects participated in the study, who were randomly assigned to either to a NF group or to an active (placebo-based) control group. The two groups performed the same experimental protocol, they only differed in that the control group received sham feedback. Ten subjects were assigned to the NF group (seven males and three females in 


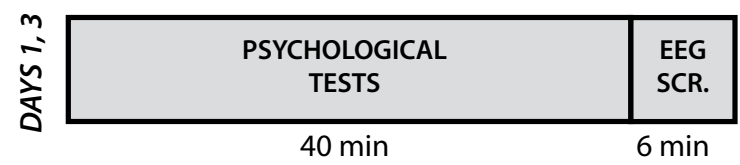

\begin{tabular}{|c|c|c|c|c|}
\hline $\begin{array}{c}\text { PRE } \\
\text { COGN. } \\
\text { ASM. }\end{array}$ & $\begin{array}{l}\text { PRE } \\
\text { EEG } \\
\text { SCR. }\end{array}$ & $\begin{array}{l}\text { NF TRAINING } \\
\text { (REAL/SHAM) }\end{array}$ & $\begin{array}{l}\text { POST } \\
\text { EEG } \\
\text { SCR. }\end{array}$ & $\begin{array}{c}\text { POST } \\
\text { COGN. } \\
\text { ASM. }\end{array}$ \\
\hline $9 \mathrm{~min}$ & $6 \mathrm{mi}$ & $25 \mathrm{~min}$ & $6 \mathrm{mir}$ & $9 \mathrm{~min}$ \\
\hline
\end{tabular}

Fig. 1. Experimental setup of the 3-day study. Day 1 and Day 3 contained a battery of psychological tests and EEG screenings. Day 2 contained the NF training (either real or sham feedback). Cognitive assessments and EEG screenings were performed pre-post the training. Note: SCR, screening; COGN.ASM, cognitive assessment.

the range $25.8 \pm 4.07$ years) and nine to the control group (seven males and two females in the range $24.3 \pm 3.67$ years). Participants were duly informed about the entire protocol of the study before signing the consent forms, and were blind to group assignment. The participants were not informed of the existence of a placebo-based control group to avoid the possible lack of motivation or effort of users thinking they were assigned to the control group [9].

The experimental setup consisted of three consecutive days (Figure 1). The NF training focused on upper alpha enhancement in parieto-occipital areas and was performed on Day 2. It consisted of 5 trials of 5 minutes each ( 25 minutes). An EEG screening and a cognitive task were interleaved immediately pre-post the training (on the same day) to assess EEG and behavioral changes. An EEG screening and a battery of psychological tests were performed on the day before and after the training (Days 1 and 3) to assess the one-day lasting EEG and behavioral changes.

EEG signals were recorded from 16 active electrodes placed at FP1, FP2, F3, Fz, F4, C3, Cz, C4, P7, P3, Pz, P4, P8, O1, Oz and O2 (10/10 system). Ground and reference electrodes were placed on FPz and the left earlobe, respectively. EEG was amplified using a commercial gTec system at a sampling rate of $256 \mathrm{~Hz}$, power-line notchfiltered at $50 \mathrm{~Hz}$, and $[0.5-60] \mathrm{Hz}$ bandpass-filtered. Signal acquisition, processing and feedback presentation were developed using Bit\&Brain Technologies software.

\section{B. NF Procedure}

1) EEG Screenings: Screenings were included to assess changes in the EEG. Each screening was comprised of a passive and an active screening. The passive screening was a 3-min recording in a closed-eyes resting state where the users were instructed to stay relaxed. The active screening was a 3-min recording in an open-eyes active state in which users faced a square on a screen that changed randomly from gray to red or from gray to blue gradually (i.e., changing saturation), and users were instructed to count the number of saturation changes from gray to red as a cognitive challenge.

2) NF Training: Training focused on the enhancement of upper alpha (UA) power for electrode locations P3, Pz, P4, $\mathrm{O} 1$ and $\mathrm{O} 2$ (these locations are referred to as feedback locations). Independent Component Analysis (ICA) was com- puted using the active screening, and blinking components were automatically removed. IAF was computed per user and feedback location as the peak frequency in the traditional alpha range of [7-13] $\mathrm{Hz}$ in the active screening. Passive screening was used to define the IAF when no clear alpha peak was found in the active screening. UA was then defined as the interval [IAF, IAF+2] Hz [3]. UA power was calculated for online operation by means of a sliding window FFT with a 1 second hamming window, $30 \mathrm{~ms}$ of overlapping, and 1024 points using zero-padding $(0.25 \mathrm{~Hz}$ resolution). Baseline was set to the averaged UA power across the feedback locations for the active screening. Feedback was updated each $30 \mathrm{~ms}$ according to the average UA power for the feedback locations. The feedback was provided visually as the participants faced a square on a screen, either red or blue according to whether the UA power was higher or lower than the baseline, respectively. The saturation of the square was proportional to the deviation of the UA power from the baseline (saturation scale covered $95 \%$ of the power values).

a) Sham Feedback: An identical procedure to the one previously described for the NF group was also followed for the control group, except for the feedback. In the control group, real feedback was computed but a registered EEG signal of another user in the same NF training protocol was used to provide them with sham feedback. All participants of the control group received the same sham feedback.

\section{Behavioral Assessments}

1) Cognitive Assessment: A mental rotation task of solid figures was performed to measure behavioral changes, and to evaluate the NF effects on the EEG during its execution. A standard spatial visualization Spanish test [10] was adapted and computerized to simultaneously acquire EEG during its execution. The test consisted of 50 trials, executed in two runs. In each trial one item consisting of two figures, a target and a test figure, arranged one above the other, was presented. Participants were asked to indicate whether the test figure was a rotated target or not, by pushing a corresponding switch. Each trial lasted $7.5 \mathrm{~s}$ and started with a fixation cross at the center of the screen for $1.5 \mathrm{~s}$. The item was then presented for $6 \mathrm{~s}$. Finally, a gray screen was displayed during the inter-trial interval (2.5 s). Participants were requested to respond as quickly and accurately as possible, starting from the apparition of the item. Total test lasted approximately $8.5 \mathrm{~min}$. Behavioral scores were the total number of correct responses and the reaction times.

2) Psychological Tests: The following battery of psychological tests was administered:

a) Paced Auditory Serial Addition Task (PASAT [11]): evaluates rate of information processing and working memory. The PASAT has demonstrated good psychometric properties, such as high levels of internal consistency and testretest reliability [12].

b) Rey Auditory Verbal Learning Test (RAVLT) [13]: evaluates retention and immediate evocation, verbal learning and remembering items after interference task and recognition. A Spanish version was administered [14]. 


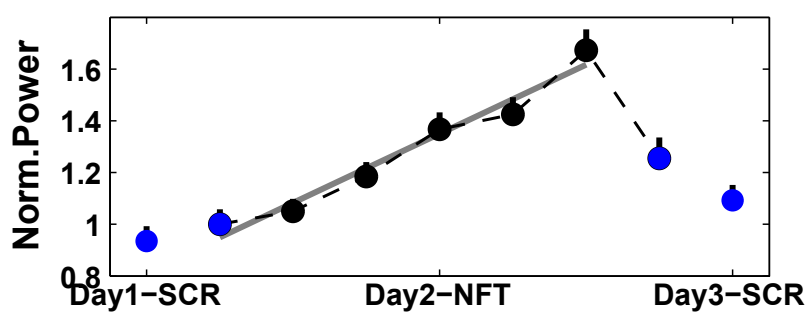

(a) NF group

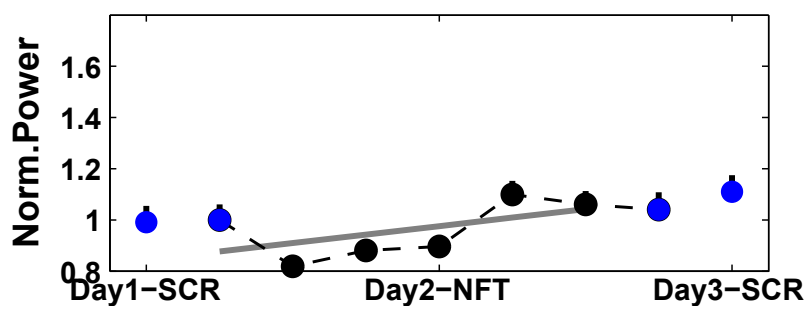

(b) Control group

Fig. 2. UA progress for each group. Each figure contains Day 1 screening, Day 2 pre-post screenings and NF trials, and Day 3 screening. Blue dots denote active screenings and black dots NF trials. The grey line shows the tendency of the pre-screening and trials. Values are normalized per user to the UA power in the Day 2 pre-active screening. Vertical bars indicate sem.

c) Trail Making Test (TMT) [15]: provides information on visual search, scanning, speed of processing, mental flexibility, and executive functions. The TMT is sensitive to a variety of neurological impairments and processes [16], [17].

d) Stroop Color-Word Test [18]: evaluates cognitive performance, attention and concentration [14], measures the individual capacity to solve cognitive stress, inhibit interferences and process complex data. This test also provides a general measure of cognitive flexibility and control or executive functioning [19].

\section{RESUlts}

This section evaluates the NF effects on UA band during the NF training, and during the execution of the cognitive assessment (subsection III-A and III-B, respectively). Then, the behavioral effects of the training are reported (subsection III-C). Statistical significance was assessed using paired onetailed t-tests for within-group comparisons, and unpaired one-tailed t-tests for between-group comparisons.

\section{A. UA Training Progress}

This section analyzes the EEG screenings and training trials to assess the NF effects on the EEG. EEG was preprocessed for offline analysis using a threshold-based filter of $250 \mu \mathrm{V}$. Then, power values were estimated using the Welch method on $50 \%$ overlapped 500 ms Hamming windows. Frequency resolution was set to $0.25 \mathrm{~Hz}$. UA power results are displayed in Figure 2. Due to the large inter-user variability in absolute power, results are normalized with the UA power in the pre-active screening of Day 2. Note that Day 2 active screening did not show statistical significant differences between groups in absolute power $\left(9.56 \mu \mathrm{V}^{2}\right.$ for $\mathrm{NF}$ group and $9.55 \mu \mathrm{V}^{2}$ for control group).
Training progress was measured by the tendency of UA power in the pre-active screening and training trials. The training progress was reflected by a significant positive tendency in that metric: the gradients of a fitted regression line for each subject of the NF group were significantly greater than zero $(t(9)=2.52, p=0.016)$. This metric was not significant for the control group, and the difference between groups was not significant. The UA increase between the preactive screening of Day 2 and the last trial was also measured to assess training progress. This increase was $67 \%$ for the $\mathrm{NF}$ group (significantly greater than zero, $t(9)=2.65, p=$ 0.013 ), and $6 \%$ for the control group (not significant). The difference between groups was significant $(t(17)=1.97, p=$ 0.032). Functional changes in the EEG were measured using the pre-post active screenings of Day 2. UA pre-post increase was $25 \%$ for the NF group (significantly greater than zero, $t(9)=4.12, p=0.001$ ), and $4 \%$ for the control group (not significant). The difference between groups was significant $(t(17)=2.42, p=0.013)$. The one-day maintenance of the UA functional changes was measured by comparing the preactive screening of Day 2 and active screening of Day 3, and no significant differences were found within groups.

The results show a significant training progress only for the NF group, which was significant between groups in one of the two metrics used. Functional changes measured immediately pre-post the training were significant for the NF group in comparison with the control group. These functional changes were not significantly maintained the next day. These results show the key role of the feedback: only the NF group, which received real feedback, was able to enhance upper alpha during the training trials, and this increase led to functional changes measured in the active screenings.

\section{B. NF Effects on UA during the Cognitive Assessment}

This section analyzes the NF effects on the EEG during the executions (pre-post the NF training) of the cognitive assessment. The objective was to study the UA time-course during the executions. The assessment was composed of two intervals: (i) rest interval, where the user was waiting for the item presentation; and (ii) task interval, where the user was mentally rotating the figures. Complete rest and task intervals lasted $1.5 \mathrm{~s}$ and $6 \mathrm{~s}$, respectively. For each group a modification of the event-related desynchronization (ERD) metric [20] was computed: the baseline for prepost NF executions was set to the averaged UA power in rest interval of the pre NF execution to allow for pre-post visual comparison. Results are shown in Figures 3a-b. A desynchronization (about 40\%) starting about $200 \mathrm{~ms}$ after figure presentation can be observed. An increase in power in the rest interval can be observed (specially for the NF group) whereas the power in the task interval remains constant.

The averaged UA power for all intervals in each group was measured to assess the statistical significance of the results. Complete rest interval was used for the subsequent analysis. A subset of the complete task interval (the $4 \mathrm{~s}$ time interval starting $1.5 \mathrm{~s}$ after the stimulus onset, which is shaded in Figures 5a-b) was defined to isolate the ERD 


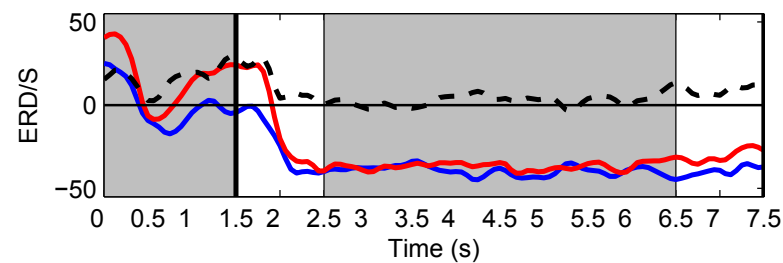

(a) NF group: UA time-course

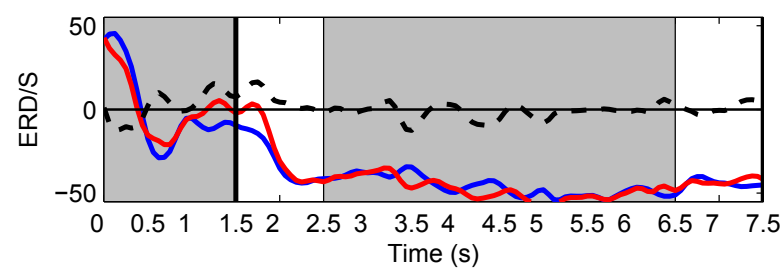

(b) Control group: UA time-course REST

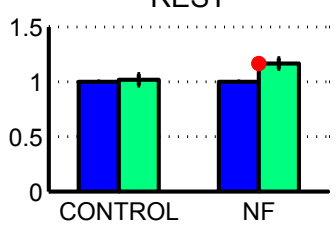

TASK

(c) Between group comparison

Fig. 3. (a,b) UA time-course ERD/S for each group in the mental rotation task. The blue and red lines show the ERD/S pre-post the NF training, respectively. The difference between them is represented by the dashed black line. Grey colored areas denote the rest and task subintervals for power measurements depicted in c. (c) Mean and sem values for the pre (blue) and post (green) power values in the rest and task intervals, for each group. A red point denote significant differences.

phenomenon. These measurements were normalized per user to the averaged UA power during the rest interval of the pre $\mathrm{NF}$ execution. Results are shown in Figure 3c.

The results show that only the NF group presented a significant increase in power $(17 \%)$ during the rest interval $(t(9)=$ $-3.44, p=0.003)$. The increase of this measurement was significant between groups $(t(17)=-2.09, p=0.026)$. The power in the task interval remained constant for both groups and no significant increase differences were found between groups. In conclusion, the NF group enhanced UA power during the NF training, which led to an increase in the rest interval while maintaining the power constant during task interval. Thus, only the NF group presented a greater desynchronization, which is hypothetized to be positively related to cognitive performance [4], [7].

\section{Behavioral Results}

This section analyzes the behavioral results of the cognitive assessment and the psychological tests.

1) Cognitive Assessment: Only responses in the complete task interval $(6 \mathrm{~s})$ plus $2.5 \mathrm{~s}$ (inter-trial interval) were taken into account. Reaction times were computed only for correct responses. Results are depicted in Figure 4. Regarding the correct responses, both groups significantly improved performance. This increase was greater for the NF group, but was not significant between groups. Similar results were obtained

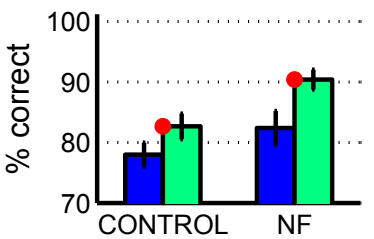

(a) Responses (within)

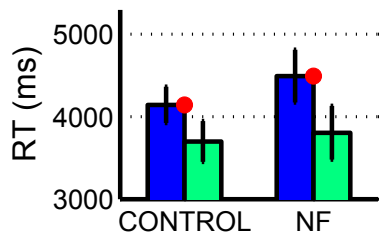

(c) RT (within)
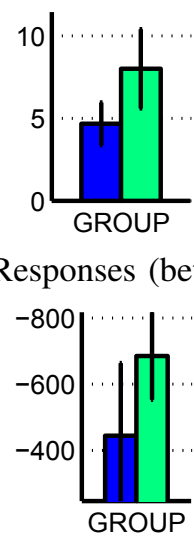

(d) RT (between) (b) Responses (between)

Fig. 4. Mean and sem values for the correct responses (upper figures) and the reaction times (lower figures). Left-side figures show within-group comparisons: the pre-post NF scores (blue and green columns, respectively) for each group. Right-side figures show the pre-post increase for the control group (blue column) and the NF group (green column).

in the reaction times measurement. Both groups significantly reduced reaction time, with the NF group performing better, but the difference between groups was not significant. These results show a strong learning effect that may have masked the changes in performance due to NF training. Furthermore, initial scores were $82.4 \%$ and $78 \%$ for the NF and control groups, respectively. The high initial scores suggest that the assessment was too simple for the participants of the study, which could be considered a methodological limitation.

2) Psychological Tests: Mean summary data and difference scores between NF and control groups are displayed in Table I. A statistically significant difference was found in the PASAT test with the NF group, both in time elapsed and errors. The control group did not show differences in error measurement; however, the differences between groups were not significant. Note that this test evaluates working memory, which is related to NF training [4]. The verbal memory was evaluated using RAVLT. A significant increase was found in the number of correct words recalled in both groups, however this increase was apparent only in the NF group in the number of correct words. Differences were not significant between groups. Regarding the TMT test, between-group differences were observed when evaluating executive function by means of the part B of the test. Participants performed better after NF training when compared to the control group. The analysis of data in the attention task (STROOP) did not reveal a significant improvement in performance in interference score. In conclusion, the results obtained in the psychological tests showed a general improvement in cognitive performance for the NF group in comparison to the control group despite some limitations, such as the learning effect due to repeated measurements and baseline differences between groups (the latter may have imposed a methodological limitation taking into account the sample size of the participants of the study). 
TABLE I

Psychological Tests

Values are mean and standard deviations. Differences significance value: $* \mathrm{p}=0.05 ; * * \mathrm{p}=0.01 ; * * * \mathrm{p}=0.001$.

\begin{tabular}{|c|c|c|c|c|c|c|c|c|c|c|}
\hline \multirow[t]{2}{*}{ Test } & \multirow[t]{2}{*}{ Cognitive function } & \multicolumn{3}{|c|}{$\mathrm{NF}$} & \multicolumn{3}{|c|}{ CONTROL } & \multicolumn{3}{|c|}{ DIFF } \\
\hline & & Pre & Post & Sign & Pre & Post & Sign & $\mathrm{NF}$ & Control & Sign \\
\hline $\begin{array}{l}\text { PASAT } \\
\text { Time } \\
\text { Errors }\end{array}$ & $\begin{array}{l}\text { Working memory } \\
\text { Processing speed }\end{array}$ & $\begin{array}{c}199.9 \pm 57.3 \\
6.7 \pm 3.3\end{array}$ & $\begin{array}{c}157.8 \pm 36.5 \\
3.6 \pm 2.4\end{array}$ & $\begin{array}{l}* * * \\
*\end{array}$ & $\begin{array}{c}174.23 \pm 59.5 \\
4.1 \pm 3.1\end{array}$ & $\begin{array}{c}140.76 \pm 46.8 \\
2.8 \pm 2.5\end{array}$ & $* *$ & $\begin{array}{c}-42.1 \pm 25.2 \\
-3.1 \pm 3.2\end{array}$ & $\begin{array}{c}-33.5 \pm 26.2 \\
-1.3 \pm 2.7\end{array}$ & \\
\hline $\begin{array}{l}\text { RAVLT } \\
\text { Correct words } \\
\text { Recognition words }\end{array}$ & $\begin{array}{l}\text { Verbal memory } \\
\text { Learning with } \\
\text { interference }\end{array}$ & $\begin{array}{c}13.9 \pm 1.3 \\
71.9 \pm 18.9\end{array}$ & $\begin{array}{c}14.8 \pm 0.6 \\
88.8 \pm 14.1\end{array}$ & $\begin{array}{c}* \\
* * *\end{array}$ & $\begin{array}{c}13.6 \pm 1.6 \\
72.7 \pm 12.9\end{array}$ & $\begin{array}{c}14.5 \pm 0.7 \\
88.8 \pm 10.5\end{array}$ & $* * *$ & $\begin{array}{c}0.9 \pm 1.1 \\
16.9 \pm 7.5\end{array}$ & $\begin{array}{c}0.8 \pm 1.6 \\
16.1 \pm 8.9\end{array}$ & \\
\hline $\begin{array}{l}\text { TMT } \\
\text { TMT-A Time } \\
\text { TMT-B Time }\end{array}$ & $\begin{array}{l}\text { Executive functions } \\
\text { Attention and } \\
\text { concentration (TMT-A) }\end{array}$ & $\begin{array}{l}27.2 \pm 11.3 \\
53.9 \pm 12.9\end{array}$ & $\begin{array}{c}21 \pm 7.1 \\
36.7 \pm 14.8\end{array}$ & $* *$ & $\begin{array}{c}27 \pm 11.7 \\
36.1 \pm 10.6\end{array}$ & $\begin{array}{l}20.4 \pm 6.2 \\
28.8 \pm 7.6\end{array}$ & $\begin{array}{l}* \\
* *\end{array}$ & $\begin{array}{c}-6.2 \pm 9.6 \\
-17.2 \pm 12.8\end{array}$ & $\begin{array}{l}-6.6 \pm 8.1 \\
-7.3 \pm 6.2\end{array}$ & $*$ \\
\hline $\begin{array}{l}\text { STROOP } \\
\quad \text { Interference }\end{array}$ & $\begin{array}{l}\text { Attention and } \\
\text { concentration } \\
\text { Executive functions }\end{array}$ & $3.2 \pm 6.1$ & $6.8 \pm 11.9$ & & $10.54 \pm 9.2$ & $13.23 \pm 8.8$ & & $3.6 \pm 11.6$ & $2.7 \pm 4.1$ & \\
\hline
\end{tabular}

\section{CONCLUSIONS}

This paper presented a single-session NF training procedure of the individual upper alpha frequency. The doubleblind nature of the study with a placebo-based control group allowed for minimization of non-specific factors. Training progress and functional changes were obtained in the EEG only for the NF group, thus showing the importance of the feedback in the NF procedure. These changes in the upper alpha power for the NF group led to an increase in the upper alpha desynchronization during the execution of the cognitive assessment (not visible for the control group). Note that this desynchronization is suggested to be related to cognitive performance [4], [7]. Regarding behavioral effects, the NF group performed better in the two scores of the cognitive assessment, but no significant differences were found with the control group. The results of the psychological tests showed a general improvement in cognitive performance of the NF group when compared to the control group, although significance was only reached for one test (part B of TMT test). The obtained behavioral results may be explained by the fact that it was a strong learning effect due to repeated measurements, and baseline differences between groups in some tests. In addition to that, only 25 minutes of training may have been insufficient to achieve significant differences between groups. Also, the authors presented the results of all participants, i.e., participants of the NF group have not been classified into responders and non-responders since it would have originated unfair comparisons between groups with regard to EEG patterns. In the near future, the authors consider it interesting to replicate this study redesigning the behavioral assessments, by better adjusting the difficulty of the cognitive task to the participants of the study, and using test-retest measurements in psychological tests.

\section{ACKNOWLEDGEMENTS}

The authors want to thank Marta Puebla and Mayte Navarro for their support in the development of the study.

\section{REFERENCES}

[1] G. G Knyazev, "Motivation, emotion, and their inhibitory control mirrored in brain oscillations.," Neuroscience and biobehavioral reviews, vol. 31, no. 3, pp. 377-95, Jan. 2007.
[2] E. Basar and B. Güntekin, "A review of brain oscillations in cognitive disorders and the role of neurotransmitters.," Brain research, vol. 1235 , pp. 172-93, Oct. 2008.

[3] W. Klimesch, "EEG alpha and theta oscillations reflect cognitive and memory performance: a review and analysis," Brain Research Reviews, vol. 29, no. 2-3, pp. 169 - 195, 1999.

[4] W. Klimesch, P. Sauseng, and S. Hanslmayr, "EEG alpha oscillations: the inhibition-timing hypothesis.," Brain research reviews, vol. 53, no. 1, pp. 63-88, Jan. 2007.

[5] C. Escolano, M. Aguilar, and J. Minguez, "EEG-based upper alpha neurofeedback training improves working memory performance," in Int. Conf. Engineering in Medicine and Biology Society, USA, 2011.

[6] B. Zoefel, R. J. Huster, and C. S. Herrmann, "Neurofeedback training of the upper alpha frequency band in EEG improves cognitive performance," NeuroImage, vol. 54, no. 2, pp. 1427 - 1431, 2011.

[7] S. Hanslmayr, P. Sauseng, M. Doppelmayr, M. Schabus, and W. Klimesch, "Increasing individual upper alpha power by neurofeedback improves cognitive performance in human subjects," Applied Psychophysiology and Biofeedback, vol. 30, pp. 1-10, 2005.

[8] D. J. Vernon, "Can neurofeedback training enhance performance? An evaluation of the evidence with implications for future research," App. Psychophysiology and Biofeedback, vol. 30, pp. 347-364, 2005.

[9] H. N. Logemann, M. M Lansbergen, T. W. Van Os, K. B. Böcker, and J. L. Kenemans, "The effectiveness of EEG-feedback on attention, impulsivity and EEG: a sham feedback controlled study.," Neuroscience letters, vol. 479, no. 1, pp. 49-53, July 2010.

[10] M Yela, Rotación de figuras macizas, TEA Ediciones, 1968.

[11] D. M. A Gronwall, "Paced auditory serial-addition task: a measure of recovery from concussion," Perceptual and motor skills, vol. 44, no. 2, pp. 367-373, 1977.

[12] T. N. Tombaugh, "A comprehensive review of the Paced Auditory Serial Addition Test (PASAT)," Archives of Clinical Neuropsychology, vol. 21, no. 1, pp. 53-76, 2006.

[13] A. Rey, L'examen clinique en psychologie., Presses Universitaries de France, 1958.

[14] M. D. Lezak, D. B. Howieson, and D. W. Loring, Neuropsychological assessment, Oxford University Press, New York, 2004.

[15] R. M. Reitan, "Validity of the Trail Making Test as an indicator of organic brain damage," Perceptual and motor skills, vol. 8, no. 3, pp. 271-276, 1958

[16] M. D. Lezak, "Neuropsychology assessment," Neuropsychology assessment, 1995.

[17] T. N. Tombaugh, "Trail Making Test A and B: normative data stratified by age and education," Archives of Clinical Neuropsychology, vol. 19, no. 2, pp. 203, 2004.

[18] J. R. Stroop, "Studies of interference in serial verbal reactions.," J. of Experimental Psychology: General, vol. 121, no. 1, pp. 15, 1992.

[19] R. G. Moering, J. A. Schinka, J. A. Mortimer, and A. B. Graves, "Normative data for elderly African Americans for the Stroop color and word test," Archives of Clinical Neuropsychology, vol. 19, no. 1 , pp. 61-71, 2004

[20] G. Pfurtscheller and F. H. Lopes da Silva, "Event-related EEG/MEG synchronization and desynchronization: basic principles," Clinical Neurophysiology, vol. 110, no. 11, pp. 1842-1857, Nov. 1999. 